\title{
Maternal age and reproductive function in female Sprague-Dawley rats
}

\author{
Misawa Ishii ${ }^{1,2,3}$, Toshiaki Yamauchi², Kiyoshi Matsumoto², Gen Watanabe ${ }^{1,3}$, \\ Kazuyoshi Taya ${ }^{1,3}$ and Fumio Chatani ${ }^{2}$

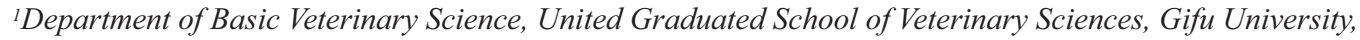 \\ Gifu 501-1193, Japan \\ 2Drug Safety Research Laboratories, Pharmaceutical Research Division, Takeda Pharmaceutical Company Limited, \\ Kanagawa 251-8555, Japan \\ ${ }^{3}$ Laboratory of Veterinary Physiology, Department of Veterinary Medicine, Faculty of Agriculture, \\ Tokyo University of Agriculture and Technology, Tokyo 183-8509, Japan
}

(Received December 21, 2011; Accepted March 9, 2012)

\begin{abstract}
We investigated the age-related changes in reproductive function in female rats of the $\mathrm{Crl}: \mathrm{CD}(\mathrm{SD})$ strain. The estrous cycles were monitored from 6 to 42 weeks of age. Other females were mated with males at $6,8,10,15,19,23,27,31,35$ and 40 weeks of age and caesarean section was performed on day 20 of gestation to examine their fetuses, and then reproductive parameters were calculated and fetal growth was observed. The percentage of rats exhibiting irregular estrous cycles increased from 23 weeks of age. The copulatory and fertility indices decreased from 35 and 27 weeks of age, respectively. Dams at 6 weeks of age showed low numbers of corpora lutea and implantation sites. Although the number of corpora lutea was not affected by advanced maternal age, number of implantation sites decreased from 27 weeks of age. The pre- and post-implantation loss rates increased from 23 and 31 weeks of age, respectively, and the number of live fetuses decreased from 27 weeks of age. Fetal growth retardation was observed in maternal rats older than 31 weeks of age. The external observations on the fetuses revealed umbilical hernia at 35 to 40 weeks of age. These data indicated that maternal aging affected reproductive function and fetal development from 23 and 31 weeks of age, respectively. It was considered that the appropriate age of $\mathrm{Crl}: \mathrm{CD}(\mathrm{SD})$ female rats for the assessment of reproductive toxicity were 8 to 22 weeks.
\end{abstract}

Key words: Aging, Reproductive parameter, $\mathrm{Crl}: \mathrm{CD}(\mathrm{SD})$ rat, Embryonic/fetal development

\section{INTRODUCTION}

Elder women and female mammals show a decline in reproductive function with irregular menstrual and estrous cycles, ovulatory disturbance and decrease in fertility (Cooper et al., 1980; Dunson et al., 2004; Matt et al., 1998; Nass et al., 1984; Weiss et al., 2004). Rats are widely used in reproductive and developmental toxicity studies to evaluate the effects of pharmaceuticals and chemicals on the estrous cycle, fertility and the development of embryos/fetuses. Therefore, an appropriate age of the female rats should be selected for reproductive and developmental toxicity studies. However, the guideline for reproductive and developmental toxicity study does not specify the age of the animals (ICH Steering Committee,
1993, 1994, 1995; OECD, 2001a, 2001b). Although the decline in reproductive function with advance in maternal age should be considered, there are few studies that have investigated the precise age-related changes in the reproductive parameters and fetal growth. Previous studies reported that a small litter size, low fertility and suppressed embryonic/fetal viability were observed in middle-age rats of Wistar strain (Lapolt et al., 1986; Matt et al., 1987). However, these reports focused on only the viability of the embryos/fetuses and no reports concerning estrous cycles and mating throughout their conceptuses were found.

In the present study, we used nulliparous SpragueDawley rats $(\mathrm{Crl}: \mathrm{CD}(\mathrm{SD})$ rats), which is the most common strain for reproductive toxicity study and investi-

Correspondence: Misawa Ishii (E-mail: Ishii_Misawa@takeda.co.jp) 
gated how increasing maternal age affected reproductive function including the estrous cycles, mating and embryo/ fetal development. The estrous cycles of female SD rats were monitored from 6 through 42 weeks of age and the changes in the reproductive parameters were examined including copulation and fertility indices, number of corpora lutea and implantation sites, pre- and post-implantation loss rates, number of live fetuses, and fetal growth from 6 to 40 weeks of maternal age. External, visceral and skeletal observations were also performed for their live fetuses. According to these data, we evaluated the approximate age for reproductive and toxicity studies.

\section{MATERIALS AND METHODS}

\section{Animals}

Female Crl:CD(SD) rats, obtained from Charles River Japan, Inc. (Kanagawa, Japan) at 5 or 8 weeks of age, were housed individually under lighting conditions of a 12-hr light/dark cycle (lights on from 7:00 to 19:00). The animals were allowed free access to tap water and a laboratory animal diet (CR-LPF, $\gamma$-ray irradiated, Oriental Yeast Co. Ltd., Tokyo, Japan) and were acclimated for at least 1 week prior to the experiments. All animal studies were performed in accordance with the institutional guidelines for animal care at Takeda Pharmaceutical Company Limited.

\section{Determination of the estrous cycles}

Vaginal smears were monitored daily in the morning from 6 weeks to 42 weeks of age $(\mathrm{N}=48)$. The vaginal smears, collected using a swab, were stained with Giemsa's solution after ethanol fixation and examined for signs of abnormalities in the estrous cycles. The types of estrous cycles were classified as follows: normal estrous cycle (4- or 5-day estrous cycle) and irregular estrous cycles including prolonged estrous cycle (6-day or longer estrous cycle), persistent estrus (cornified cells were predominant in the daily vaginal smears) and persistent diestrus (continuous diestrus phase). Estrous cycles were evaluated for 2 weeks at 6-7, 8-9, 9-10, 11-12, 16-17, 21-22, 23-24, 26-27, 31-32, 36-37 and 41-42 weeks of age.

\section{Study for mating ability, fertility and embryo/fetal development}

Mating was performed at 6, 8, 10, 15, 19, 23, 27, 31, 35 and 40 weeks of age $(\mathrm{N}=10$ for $6-27$ weeks and $\mathrm{N}=$ 20 for 31-40 weeks of age) after monitoring the estrous cycles for 1 week. Females were mated on a one-to-one basis with males for up to a maximum of 1 week. The females were examined daily for the presence of a copulation plug and the day on which the plug was found was designated as day 0 of gestation. All the dams were sacrificed by exsanguination under carbon dioxide anesthesia and then necropsied on day 20 of gestation. The numbers of corpora lutea and implantation sites and the numbers of dead embryos/fetuses (resorptions, placental remnants and dead fetuses) and live fetuses were counted for animals that were pregnant. The live fetuses were weighed and examined for external abnormalities. For the visceral observation, approximately half of the live fetuses were injected with a saturated magnesium chloride in 10 vol\% neutral buffered formalin solution into the thoracic cavity, and fixed in 10 vol\% neutral buffered formalin. Fetuses were examined for visceral abnormalities and variations according to Barrow's and Wilson's techniques (Barrow and Taylor, 1969; Wilson, 1965). The remaining live fetuses in each litter were eviscerated, fixed in 99 vol\% ethanol and stained with alizarin red $\mathrm{S}$ by a modified Dawson's technique (Dawson, 1926). The fetuses were examined for skeletal abnormalities and variations. In addition, the degree of ossification was examined by counting the sacro-caudal vertebrae. All observations on the fetuses were conducted using a dissecting microscope.

\section{Statistics and evaluation of data}

For each examination, the data for each age were compared to those at 10 weeks of age as the control. The data were tested by Bartlett's test for homogeneity of variance. Estrous cycles, copulation and fertility indices, the numbers of corpora lutea and implantation sites, the pre- and post-implantation loss rates, numbers of live fetuses and the numbers of ossified sacro-caudal vertebrae were tested by the non-parametric Steel's test because the variances were not homogenous. Body weights of fetuses obtained at these maternal ages were tested by the parametric Dunnett's test because the variances were homogenous. The statistical analysis was performed using the SAS function PROBMC (SAS/STAT Software: Changes and Enhancements, through Release 6.11. SAS Institute Inc., Cary, NC, USA). All parameters were also compared to the historical data in our laboratory.

\section{RESULTS}

\section{Estrous cycle}

At 9-10 weeks of age, all 48 females showed normal estrous cycles. There was no significant differences between 9-10 weeks of age and any other age. However, the percentages of rats exhibiting normal estrous cycles 
Maternal age and reproductive function

at ages older than 23-24 weeks of age were lower than the minimum value in the historical data in our laboratory (90\%). The percentage of rats exhibiting normal estrous cycles at 23-24 weeks of age was $89 \%$ and this reached $35 \%$ at $41-42$ weeks of age. The main type of irregular estrous cycles was prolonged estrous cycle observed at 23-24 weeks of age while persistent estrus became frequent after 26-27 weeks of age followed by persistent diestrus. The frequency of normal estrous cycles, prolonged estrous cycles, persistent estrus and persistent diestrus at 41-42 weeks were approximately 35, 25, 5 and $35 \%$, respectively (Fig. 1).

\section{Reproductive parameters}

In the animals that were necropsied on day 20 of gestation, $100 \%$ of females showed normal estrous cycles at 10 weeks of age. Rats older than 31 weeks of age showed a lower percentage of normal estrous cycles (75\%) than the minimum value in the historical data in our laboratory $(90 \%)$, and the percentage of rats exhibiting normal

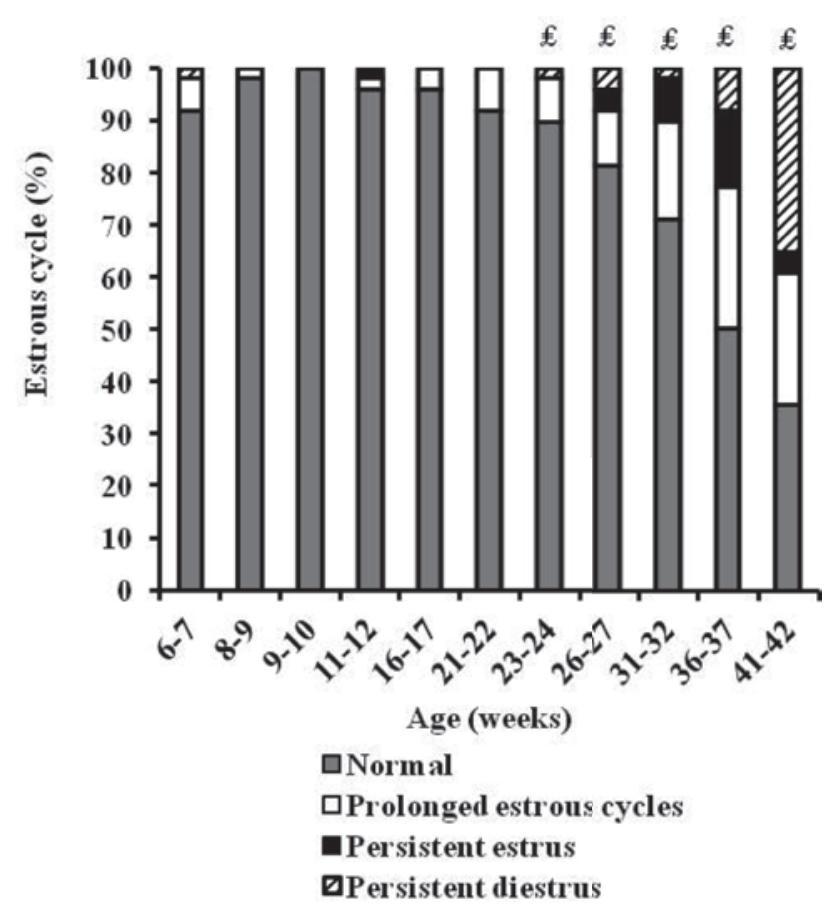

Fig. 1. Maternal age and estrous cycle pattern. Estrous cycles were monitored at $6-42$ weeks of age $(\mathrm{N}=48)$. Estrous cycles were judged as normal ( $\square, 4$ - or 5-day estrous cycles), prolonged estrous cycles ( $\square, 6$-day or longer estrous cycles), persistent estrus ( $\square$, cornified cells were predominant in the daily vaginal smears) or persistent diestrus ( $\mathbb{Z}$, continuous diestrus phase). £: outside the historical data in our laboratory. estrous cycles at 40 weeks of age was $65 \%$ (Fig. 2A). The copulation and fertility indices at 10 weeks of age were $100 \%$. There were no significant differences in dams at any age when compared to dams in 10 weeks of age. However, the copulation index in dams older than 35 weeks of age and the fertility index in dams older than 27 weeks of age were lower than the minimum value in historical data in our laboratory (copulation index: 90.0\%, fertility index: $90.0 \%$ ). The copulation index at 35 weeks of age was $80 \%$ and the fertility index at 27 weeks of age were $78 \%$, and these parameters decreased to $70 \%$ at 40 weeks of age (Fig. 2B). The numbers of corpora lutea and implantation sites at 10 weeks of age were 15.7 and 14.6, respectively, and these parameters were not significantly different between dams at 10 weeks of age and dams of any other age. However, the numbers of corpora lutea at 6 weeks of age (13.9) were lower than the minimum value in the historical data in our laboratory (14.4) and this parameter was not affected by advanced maternal age. The numbers of implantation sites at 6 weeks of age and older than 27 weeks of age were lower than the minimum values in historical data in our laboratory (13.5). The numbers of implantation sites at 6 and 27 weeks of age were 12.3 and 12.6 , respectively, and decreased to 10.4 at 40 weeks of age (Fig. 2C).

\section{Caesarean sections (Embryo/fetuses development)}

The pre- and post-implantation loss rates at 10 weeks of age were $8 \%$ and $9 \%$, respectively. There were no significant differences between the dams at 10 weeks of age and those at any other age. However, the pre-implantation loss rates in dams older than 23 weeks, except at 35 weeks and the post-implantation loss rates in dams older than 31 weeks were higher than the maximum value in the historical data in our laboratory (pre-implantation loss rate: $14.2 \%$, post-implantation loss rate: $10.9 \%$ ). The pre-implantation loss rate at 23 weeks of age was $20 \%$, and increased to $30 \%$ at 40 weeks of age (Fig. 3A). The post-implantation loss rate at 31 weeks of age was $25 \%$ and increased to $40 \%$ at 40 weeks of age (Fig. 3B). The number of live fetuses at 10 weeks of age was 13.0, and that was significantly higher than that at 40 weeks of age. Further, this parameter at 6 weeks of age and older than 27 weeks was lower than that minimum value in historical data in our laboratory (12.9). The numbers of live fetuses at 6 and 27 weeks of age were 12.0 and 11.4, respectively and this decreased to 5.9 at 40 weeks of age (Fig. 3C).

\section{Fetal growth and morphological abnormality}

Body weights for the male and female fetuses at 10 
A
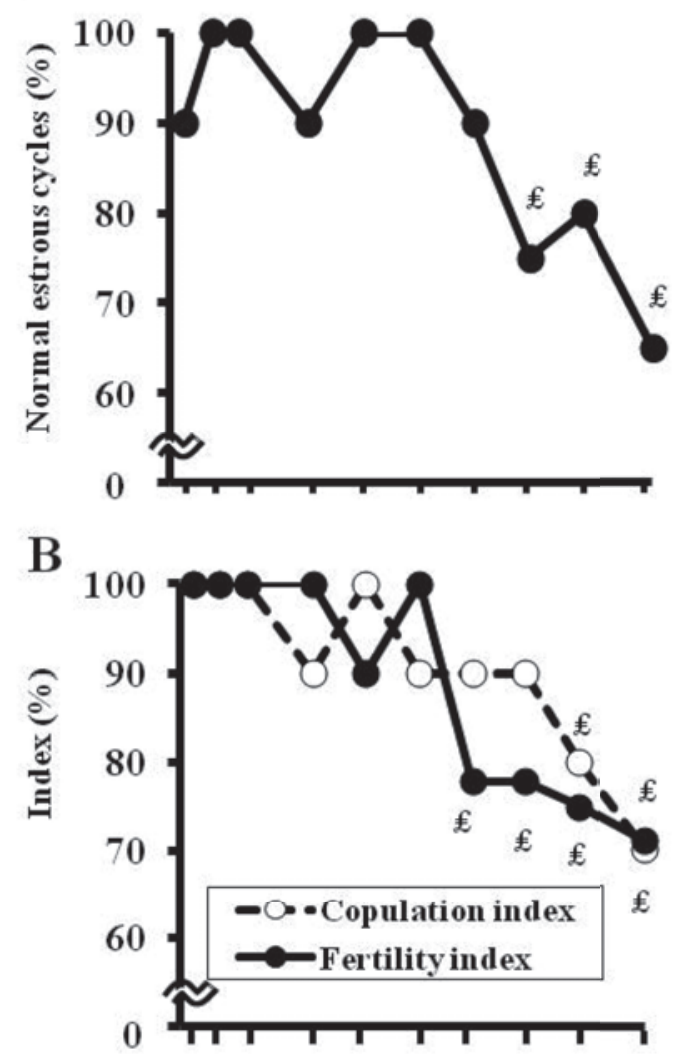

C

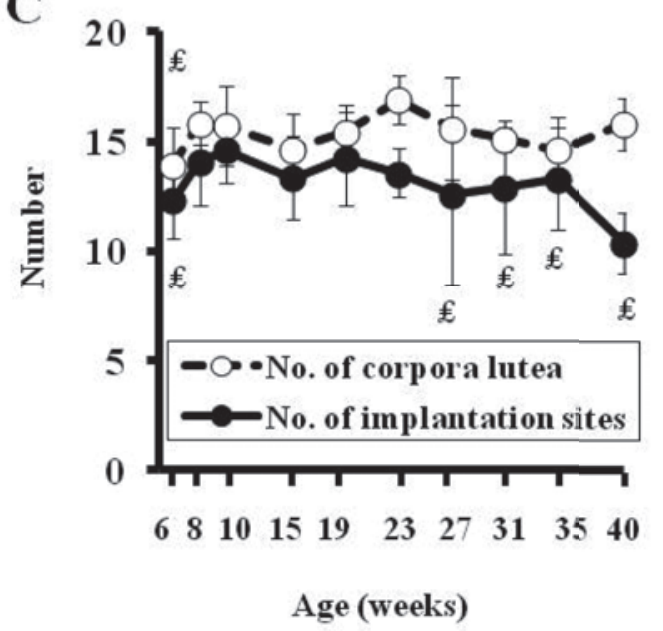

Fig. 2. Maternal age and reproductive parameters. Females of 6-40 weeks of age were mated with adult males and necropsied on day 20 of gestation $(\mathrm{N}=10,6-27$ weeks of age; $\mathrm{N}=20,31-40$ weeks of age). A: Percentage of rats which showed normal estrous cycles before the mating (6-40 weeks of age), B: Copulation and fertility indices, C: Numbers of corpora lutea and implantation sites, Mean \pm S.D., £: outside the historical data in our laboratory.
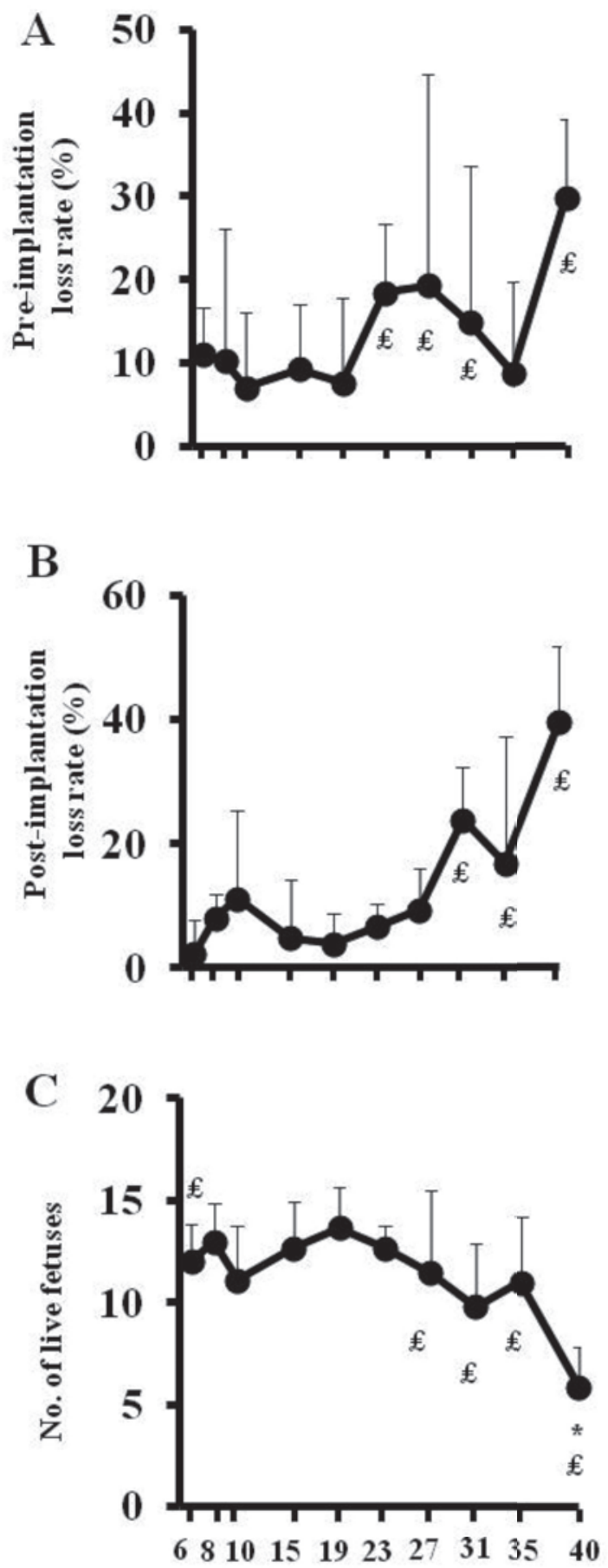

Age (weeks)

Fig. 3. Maternal age and fetal mortality. Fetuses were observed on day 20 of gestation. A: Pre-implantation loss rate, B: Post-implantation loss rate, C: Number of live fetuses. Mean \pm S.D., *: $\mathrm{P}<0.05$ vs 10 weeks of age by non-parametric Steel's test, £: outside the historical data in our laboratory. 
Maternal age and reproductive function

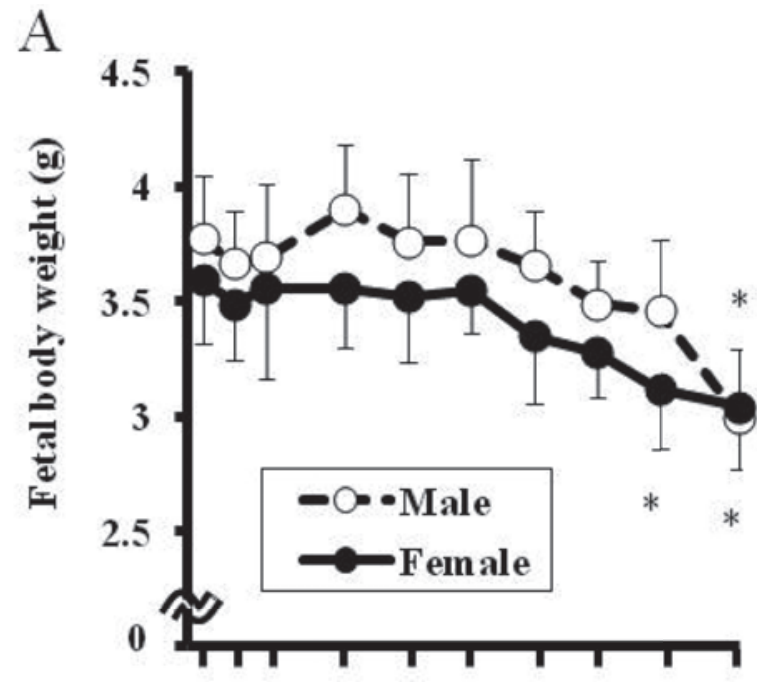

B

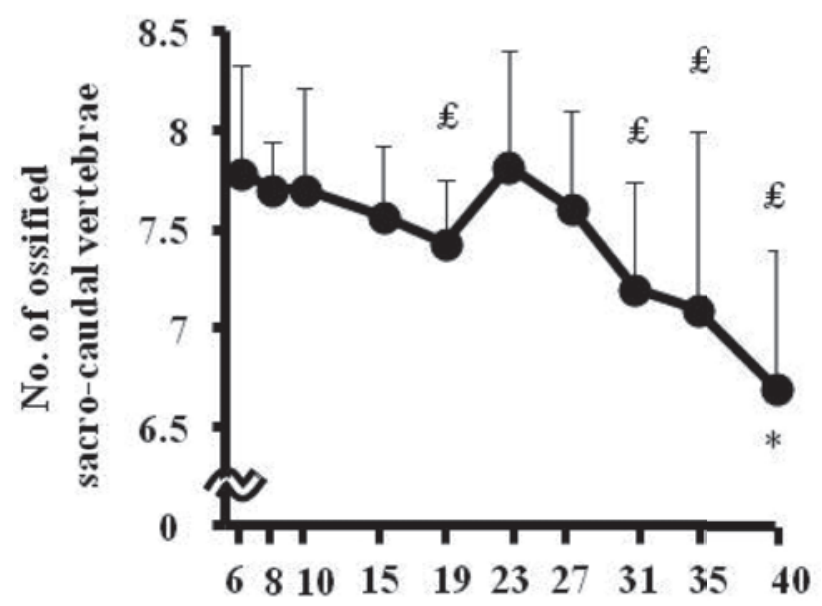

Age (weeks)

Fig. 4. Maternal age and fetal growth. A: Fetal body weight, B: Number of ossified sacro-caudal vertebrae. Mean \pm S.D., *: $\mathrm{P}<0.05$ vs 10 weeks of age by parametric Dunnett's test (A) or non-parametric Steel's test (B), £: outside the historical data in our laboratory.

weeks of maternal age were 3.67 and $3.48 \mathrm{~g}$, respectively. The body weights for male fetuses at 40 weeks of maternal age $(2.99 \mathrm{~g})$ and those in females at 35 and 40 weeks of maternal age (3.12 and 3.04, respectively) were significantly lower than those at 10 weeks of maternal age (Fig. 4A). The number of ossified sacro-caudal vertebrae at 10 weeks of maternal age was 7.7 while that at 40 weeks of maternal age (6.7) was significantly lower than that at 10 weeks of maternal age. The number of ossified sacro-cau- dal vertebrae at 19 weeks of age and older than 31 weeks of age were lower than the minimum value in the historical data in our laboratory (7.6) (Fig. 4B). The numbers of ossified sacro-caudal vertebrae at 19 and 31 weeks of age were 7.4 and 7.2, respectively. In the fetal external observations, umbilical hernia was observed in 3 of 132 (2.1\% as the total litter mean) and 1 of $59(5.6 \%)$ fetuses from different dams at 35 and 40 weeks of maternal age, respectively. The incidences were outside the historical data in our laboratory (maximal value $0.6 \%$ ). In the visceral observations, abnormalities and variations of the cardiovascular system were observed sporadically; these findings were not related to the maternal age. Cervical rib was observed at 31 and 40 weeks of maternal age (2.4 and $2.9 \%$, respectively) (Table 1 ); the finding was not considered to be related to the maternal age because the frequency of the finding was within the historical data in our laboratory (maximal value 6.5\%).

\section{DISCUSSION}

The data in the present study demonstrated that increasing female/maternal age affected ovarian reproductive function and the growth and development of the embryos/fetuses in SD rats. The frequencies of females with irregular estrous cycles were increased at older than 23-24 weeks of age. The copulation and fertility indices decreased at older than 35 and 27 weeks of age, respectively. Females at 6 weeks of age showed low numbers of corpora lutea and implantation sites. The numbers of corpora lutea did not change with advancing maternal age but the number of implantation sites decreased in dams older than 27 weeks of age. The pre- and post-implantation loss rates increased in dams older than 23 and 31 weeks of age, respectively. Decreased numbers of live fetuses was observed in dams older than 27 weeks of age. Fetal growth retardation was observed in dams older than 31 weeks of age, and external abnormalities including umbilical hernia were observed at older than 35 weeks of age.

Our study results for the estrous cycles were almost the same as in other reports describing that $50 \%$ of Long-Evans rats showed irregular estrous cycles at 8 months or older (Lapolt et al., 1986; Matt et al., 1987). Watanabe et al. (1994) reported that more than $80 \%$ of Jcl:Wistar rats maintained normal estrous cycles at 40 weeks of age while only $22 \%$ of $\mathrm{Crl}: \mathrm{CD}(\mathrm{SD})$ rats maintained normal estrous cycles at the same age as Jcl:Wistar rats. It is known that vaginal smears reflect the condition of the ovaries (Montes and Luque, 1988), and $\mathrm{Lu}$ et al. (1979) reported that females with persistent estrus 
M. Ishii et al.

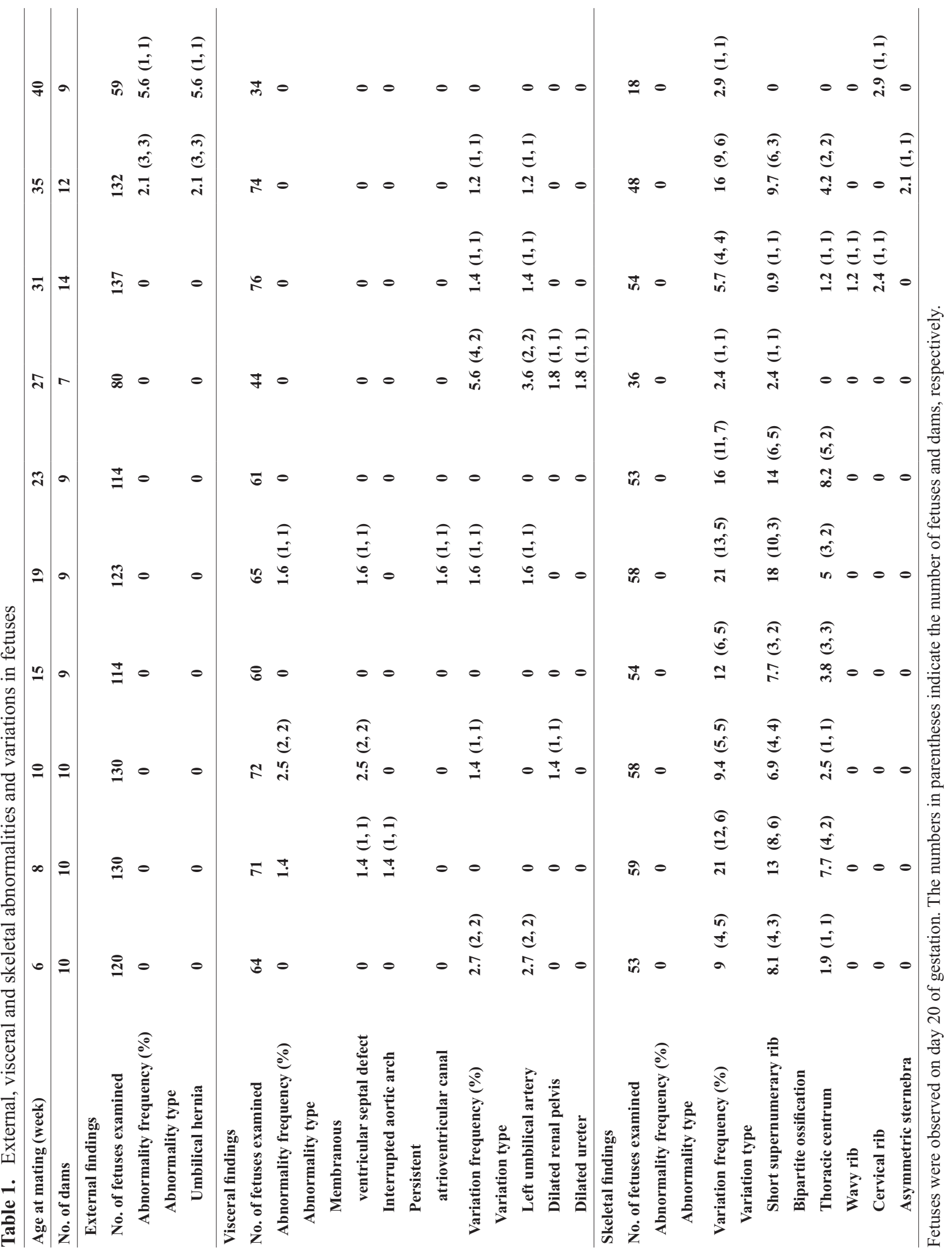

Vol. 37 No. 3 
Maternal age and reproductive function

and diestrus showed ovarian atrophy histologically and a decreased numbers of corpora lutea, indicating ovulatory disturbance. From these, it could be considered that the ovarian function in the rat declined from 23 weeks of age in female SD rats.

Females older than 35 weeks of age showed decreases in the copulation index. They also showed smaller numbers of vaginal plugs (data not shown), suggesting a low frequency of lordosis, a behavior that allows mating in female rodents; the detailed mechanism for the decrease in the numbers of vaginal plugs was unclear. The decreases in the copulation indices in middle aged females might be due to the low frequency of lordosis.

The fertility index and numbers of live fetuses decreased with advancing maternal age although the numbers of corpora lutea were not decreased. Therefore, the decreases in the fertility index were considered to be due to the decreases in the number of implantation sites as evidenced by the increases in pre-implantation loss rates. It was also considered that the decreases in the numbers of live fetuses were due to increases in the pre- and post-implantation loss rates. The number of dead fetuses was not affected by advancing age of the dams (data not shown), indicating that embryonic deaths occurred at the early or middle period of pregnancy. In nulliparous Long-Evans rats at 10 months of age, the fertility index and number of live fetuses were 29\% and 3.7 respectively (Lapolt et al., 1986). Matt et al. (1987) reported that the decline of litter size in middle-age rats was directly related to decreased numbers of normally developed blastocysts. An in vitro study showed a higher rate of cleavage delay, fragmentation or degeneration of embryos at 9-10 months of maternal age than those at 3-4 months of age (Peluso et al., 1983). The high embryonic or fetal mortality (increased pre- and post-implantation loss rates, decreased fertility index and decreased number of live fetuses) noted in the present study is probably due to a higher rate of abnormal embryos related to advancing maternal age. At 6 weeks of age, the number of live fetuses was slightly lower than the minimum values in historical data in our laboratory, and this was considered to be due to low number of corpora lutea because pre- and postimplantation loss rates did not decrease.

In the present study, the number of ossified sacro-caudal vertebrae at 19 weeks of age was lower than the minimum value in the historical data in our laboratory. It has been reported that decreased fetal body weight was one of the causes of decreased numbers of ossified sacro-caudal vertebrae, an indicator for fetal growth (Ariyuki et al., 1982). Fetal growth at 19 weeks of age was not considered to be abnormal because the degree of the decrease in the numbers of ossified sacro-caudal vertebrae was slight and the fetal body weights were not decreased. It was concluded that fetal growth retardation was occurred in dams older than 31 weeks of age. In the external observations, the increased incidence of fetal umbilical hernia at 35 and 40 weeks of age was also considered to be related to the maternal age. However, the mechanism of the development of umbilical hernia is not clear. Investigation of rat fetuses from dams older than 40 weeks of age may give information on the mechanism of age-related fetal malformations.

In summary, the present study demonstrated that advancing age of the female rats affected the following reproductive parameters: estrous cycles, the copulation and fertility indices, the number of live fetuses, embryonic/fetal growth and development. Changes in some parameters with advancing female/maternal age were different between the different strains of rats. Our results indicate that female $\mathrm{Crl}: \mathrm{CD}(\mathrm{SD})$ rats aged 8-22 weeks is the appropriate test system for reproductive toxicity study.

\section{ACKNOWLEDGMENT}

We thank Dr. Eric Spicer for reviewing and correcting the manuscript.

\section{REFERENCES}

Ariyuki, F., Ishihara, H., Higaki, K. and Yasuda, M. (1982): A study of fetal growth retardation in teratological tests: relationship between body weight and ossification of the skeleton in rat fetuses. Teratology, 26, 263-267.

Barrow, M.V. and Taylor, W. J. (1969): A rapid method for detecting malformations in rat fetuses. J. Morphol., 127, 291-305.

Cooper, R.L., Conn, P.M. and Walker, R.F. (1980): Characterization of the LH surge in middle-aged female rats. Biol. Reprod., 23, 611-615.

Dawson, A.B (1926): A note on the staining of skeleton of cleared specimens with alizarin red S. Stain. Technol., 1, 123-124.

Dunson, D.B., Baird, D.D. and Colombo, B. (2004): Increased infertility with age in men and women. Obstet Gynecol, 103, 51-56.

ICH Steering Committee (1993): Harmonized tripartite guideline: Detection of toxicity to reproduction for medicinal products.

ICH Steering Committee (1994): Harmonized tripartite guideline: Note for guidance on toxicokinetics: The assessment of systemic exposure in toxicity studies.

ICH Steering Committee (1995): Harmonized tripartite guideline: Toxicity to male fertility: An addendum to ICH harmonized tripartite guideline on detection of toxicity to reproduction for medicinal products.

Lapolt, P.S., Matt, D.W., Judd, H.L. and Lu, J.K. (1986): The relation of ovarian steroid levels in young female rats to subsequent estrous cyclicity and reproductive function during aging. Biol. Reprod., 35, 1131-1139.

Lu, K.H., Hopper, B.R., Vargo, T.M. and Yen, S.S. (1979): Chronological changes in sex steroid, gonadotropin and prolactin secre- 
M. Ishii et al.

tions in aging female rats displaying different reproductive states. Biol. Reprod., 21, 193-203.

Matt, D.W., Gilson, M.P., Sales, T.E., Krieg, R.J., Kerbeshian, M. C., Veldhuis, J.D. and Evans, W.S. (1998): Characterization of attenuated proestrous luteinizing hormone surges in middle-aged rats by deconvolution analysis. Biol. Reprod., 59, 1477-1482.

Matt, D.W., Sarver, P.L. and Lu, J.K. (1987): Relation of parity and estrous cyclicity to the biology of pregnancy in aging female rats. Biol. Reprod., 37, 421-430.

Montes, G.S. and Luque, E.H. (1988): Effects of ovarian steroids on vaginal smears in the rat. Acta Anat (Basel), 133, 192-199.

Nass, T.E., LaPolt, P.S., Judd, H.L. and Lu, J.K. (1984): Alterations in ovarian steroid and gonadotrophin secretion preceding the cessation of regular oestrous cycles in ageing female rats. J. Endocrinol., 100, 43-50.

OECD (2001a): OECD guideline for the testing of chemicals / Section 4: Health effects, Test No. 414: Prenatal development
(E-book PDF format).

OECD (2001b): OECD guideline for the testing of chemicals / Section 4: Health effects, Test No.416: Two-generation reproduction toxicity (E-book PDF format).

Peluso, J.J., Karey, K. and Gruenberg, M.L. (1983): Capacity of fertilized ova from mature and middle-aged rats to undergo preimplantation development in vitro. Cell. Tissue Res., 229, 451-456. Watanabe, C., Shirota, M. and Nagao, T. (1994): The study of agerelated changing of estrous cycle in SD female rats. Annual Report of Hatano Research Institute, 17, 37-40.

Weiss, G., Skurnick, J.H., Goldsmith, L.T., Santoro, N.F. and Park, S.J. (2004): Menopause and hypothalamic-pituitary sensitivity to estrogen. JAMA, 292, 2991-2996.

Wilson, J.G. (1965): Methods for administering agents and detecting malformations in experimental animals. Teratology: principles and techniques, 262-277. 